DECEMBER 1993, PAGES 601-612

\title{
COMPLEX DYNAMICS IN METAL CUTTING
}

\author{
BY \\ B. S. BERGER, M. ROKNI, AND I. MINIS \\ University of Maryland, College Park, Maryland
}

\begin{abstract}
The attractor associated with a system of nonlinear differential-delay equations, arising from the Wu-Liu metal cutting model, is shown to have a noninteger pointwise dimension and positive metric entropy. Projections of the attractor onto a two-dimensional plane substantiate the existence of complex dynamics. The result suggests that certain regenerative chatter states may be chaotic.
\end{abstract}

Introduction. In [1-3] a system of coupled discontinuous ordinary differential equations, identical in form to Eq. (1), model an orthogonal metal-cutting process. The cutting force, $\mathbf{F}$, is assumed to be a discontinuous function of the depth of cut and the relative velocity between tool and cutting surface. Regenerative effects are omitted. For certain parametric ranges, the response of the system is shown to be chaotic.

In the following, $\mathbf{F}$ is given by the Wu-Liu model [4], in which regenerative effects are included. The resulting coupled system of dissipative nonlinear differential delay equations was found to have unbounded solutions, contrary to experimental evidence. Boundedness was achieved through the inclusion of cubic material damping. The resulting infinite-dimensional equations are shown to have a finite-dimensional attractor for which the information dimension $D(0)=2.65 \pm 0.01$ and metric entropy $K(0)=0.11$. Positive bounded metric entropy implies chaotic dynamics.

Two-dimensional projections of trajectories on the attractor, first constructed by M. Rokni, revealed a surface-filling geometry undergoing subharmonic, nonperiodic evolution. The results of the study suggest that the complex nature of regenerative metal-cutting vibrations may be a manifestation of chaotic dynamics.

Differential-delay equations. The continuous system consisting of cutting tool and supporting structure is modeled in [4] by a set of o.d.e. of the form

$$
m \ddot{\mathbf{y}}+b \dot{\mathbf{y}}+k \mathbf{y}=\mathbf{F}
$$

where $\mathbf{y} \in \mathbf{R}^{2}, y_{1}$ and $y_{2} \equiv$ tool deflections normal and parallel to the cutting surface respectively, and $m, b$, and $k \equiv$ mass, damping, and stiffness matrices respectively. Expressions for the cutting force F [4], when substituted into Eq. (1) 
with $\mathbf{x}_{0}=\mathbf{x}(t-\tau)$, give

$$
\dot{\mathbf{x}}=\mathbf{f}(\mathbf{x}, \mathbf{x}(t-\tau))
$$

where $\mathbf{x} \in \mathbf{R}^{4}, x_{1}=y_{1}, x_{2}=y_{2}, \dot{x}_{1}=x_{3}, \dot{x}_{2}=x_{4}, \dot{x}_{3}=f_{3}, \dot{x}_{4}=f_{4}$,

$$
\begin{aligned}
f_{3}=-a_{8} x_{1}-a_{7} x_{3} & +a_{1} x_{1}(t-\tau) \\
& +\left(x_{1}-x_{1}(t-\tau)\right)\left[a_{3}\left(x_{4}-x_{4}(t-\tau)\right)-a_{2}\left(x_{3}-x_{3}(t-\tau)\right)\right]-a_{11} x_{3}^{3},
\end{aligned}
$$

and

$$
\begin{aligned}
f_{4}=-a_{4} x_{1}-a_{10} x_{2} & -a_{9} x_{4}-a_{4} x_{1}(t-\tau) \\
& +\left(x_{1}-x_{1}(t-\tau)\right)\left[a_{6}\left(x_{4}-x_{4}(t-\tau)\right)-a_{5}\left(x_{3}-x_{3}(t-\tau)\right)\right]-a_{12} x_{4}^{3} .
\end{aligned}
$$

Numerical studies indicate that the inclusion of the cubic viscoelastic damping terms [7], $a_{11} x_{3}^{3}$ and $a_{12} x_{4}^{3}$, omitted in the model proposed in [4], eliminates unbounded solutions for parametric ranges in which physical considerations require boundedness. The presence of quadratic terms and only linear dissipative terms in Eq. (2) as given in [4] is a possible cause of the instability of the unmodified system. Conditions, satisfied by Eq. (2), for the existence, uniqueness, continuous dependence, and differentiability of solutions are given in 2.2-2.4 of [8].

The choice of integration algorithm for Eq. (2) was guided by the results of [9], which showed that the Adams-Moulton predictor-corrector possesses desirable stability characteristics for pure delay equations. All numerical solutions of Eq. (2) were computed using the Adams-Bashforth predictor and Adams-Moulton corrector both of $O\left(h^{4}\right)$ with $h=(0.2) \cdot 10^{-3}$.

In subsequent numerical studies the following physical properties are assumed: feed rate, $s_{0}=0.13 \mathrm{~mm} / \mathrm{rev}$; cutting speed, $v_{0}=3 \mathrm{~m} / \mathrm{sec}$; delay, $\tau=0.4$ sec.; shear modulus of cut material, $\alpha=6 \cdot 10^{8} \mathrm{~N} / \mathrm{m}^{2}$; equivalent mass, stiffness, and linear damping coefficients for the normal and parallel directions are $83.1 \mathrm{~kg}$ and $48.86 \mathrm{~kg}, 41.9 \cdot 10^{6} \mathrm{~N} / \mathrm{m}$ and $111.1 \cdot 10^{6} \mathrm{~N} / \mathrm{m}$, and $15 \cdot 10^{3} \mathrm{~N} \mathrm{sec} / \mathrm{m}$ and $13.3 \cdot 10^{3} \mathrm{~N} \mathrm{sec} / \mathrm{m}$, respectively; width of cut, $w=0.0052 \mathrm{~m}$; and tool nose damping coefficient, $k=0$, and cubic damping coefficients $a_{11}$ and $a_{12}=1.0 \mathrm{sec}^{2} / \mathrm{m}^{2}$. The coefficients $a_{i}$ in Eq. (2) follow from Table 1, part 2 of [4]; namely, $a_{1}=$ $0.05212 \alpha w, a_{2}=0.01545 \alpha w, a_{3}=0.00426 \alpha w, a_{4}=0.10557 \alpha w, a_{5}=$ $0.01547 \alpha w, a_{6}=0.00417 \alpha w, a_{7}=0.1805 \cdot 10^{3}, a_{8}=0.05212 \alpha w+0.5042 \cdot 10^{6}$, $a_{9}=0.2722 \cdot 10^{3}$, and $a_{10}=2.2738 \cdot 10^{6}$, and initial conditions were $\mathbf{x}=0$ for $-\tau \leq t<0$ and $\mathbf{x}=\left\{0.1 \cdot 10^{-3}\right\} \cdot I$ at $t=0$.

Qualitative analysis. The frequency $129.96 \mathrm{~Hz}$, associated with the smaller of the two eigenvalues of the linearized uncoupled form of Eq. (2), is clearly evident in the time series plots of Figs. 1 and 2 (see pp. 603 and 604). $x_{1}$ and $x_{2}$ exhibit oscillatory components with frequencies of $126.7 \mathrm{~Hz}$ and $130.6 \mathrm{~Hz}$, respectively. For $40.0 \leq$ 
$t \leq 55.0,55.0 \leq t \leq 70.0$, and $120.0 \leq t \leq 135.0$, the corresponding max and min frequencies in $x_{1}$, computed as averages over fifty cycles, are $(129.5 \mathrm{~Hz}, 126.9 \mathrm{~Hz})$, $(127.0 \mathrm{~Hz}, 126.7 \mathrm{~Hz})$, and $(131.1 \mathrm{~Hz}, 126.8 \mathrm{~Hz})$, respectively.

The amplitudes of $x_{1}$ and $x_{2}$ are strongly modulated by a spectrum of lowfrequency oscillations. This is particularly evident in $\max \left(x_{1}\right)$ for $40.0 \leq t \leq 163.0$, over the compressed time scale of Fig. 3 (see p. 604).

Figures 4-10 (see pp. 605-608) are projections of the system's attractor onto the $\left(x_{3}, x_{4}\right)$ phase plane for $15 \mathrm{sec}$ intervals with $40.0 \leq t \leq 195.0$. The positions of the outer boundaries of all figures are identical. However, the geometry of trajectories over the attractor varies qualitatively (see, e.g., Figs. 4 and 7). The similarity between Figs. 4 and 10 and between Figs. 6 and 9 suggests that over the attractor the trajectory geometry undergoes a subharmonic evolution. The projection of the attractor onto the $\left(x_{1}, x_{2}\right)$ - and $\left(x_{2}, x_{4}\right)$-planes is shown for $80.0 \leq t \leq 95.0$ in Figs. 11 and 12 (see pp. 608 and 609). These are seen to have surface characteristics that are identical to the corresponding $\left(x_{3}, x_{4}\right)$ projection. Superposition of sequences of the projections yields a surface that suggests the presence of chaotic dynamics.

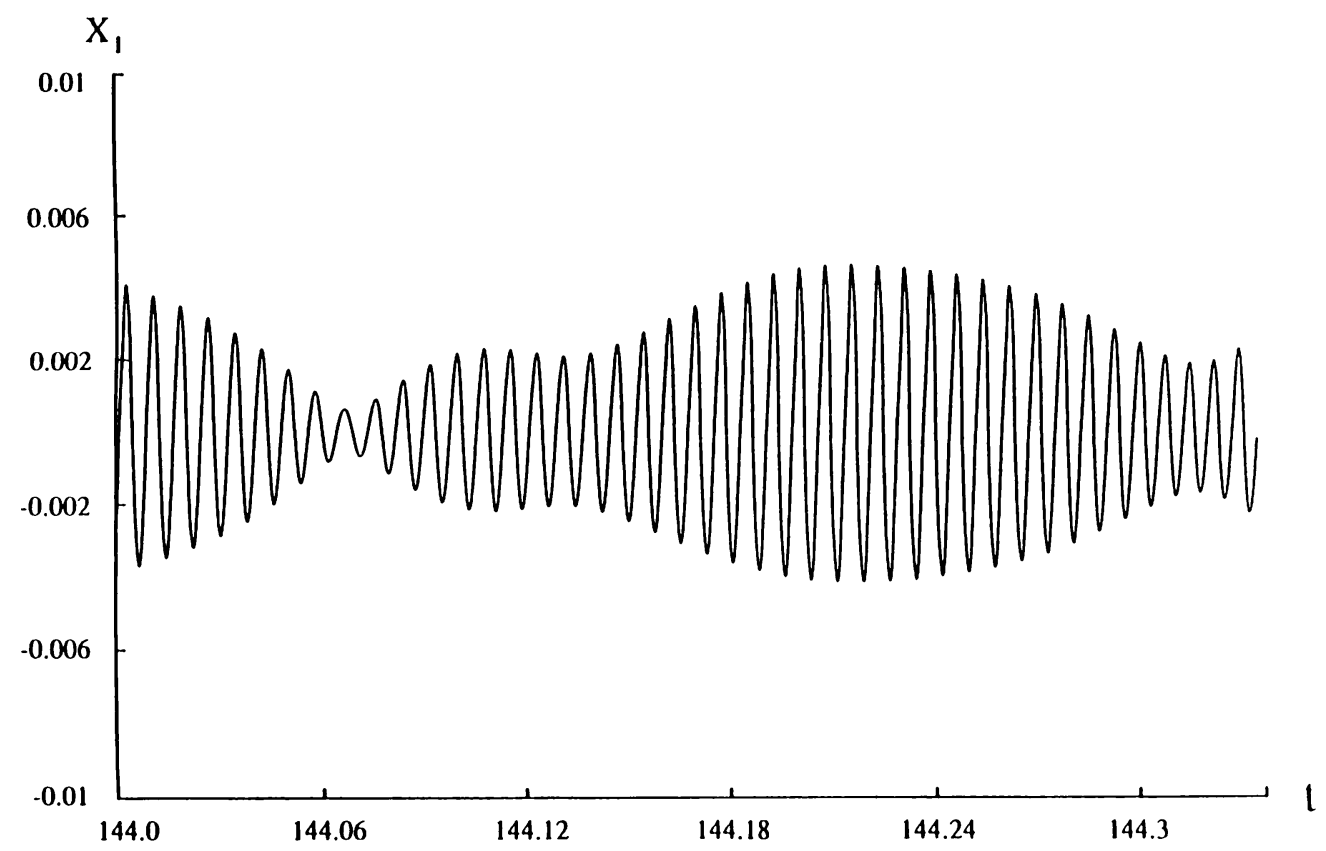

Fig. 1. $x_{1}$ vs. $t$ for $144 \leq t \leq 144.3$. 


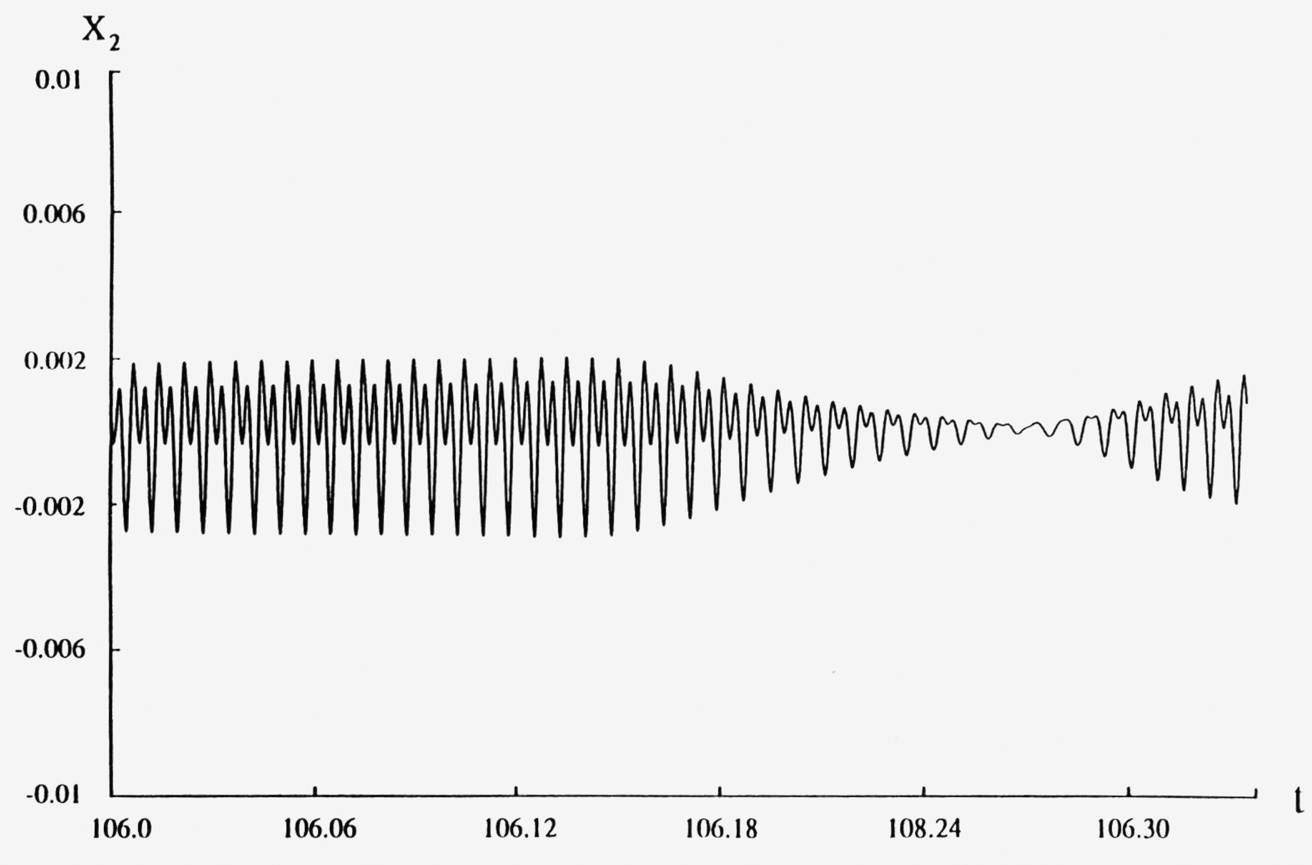

Fig. 2. $x_{2}$ vs. $t$ for $106 \leq t \leq 106.3$.

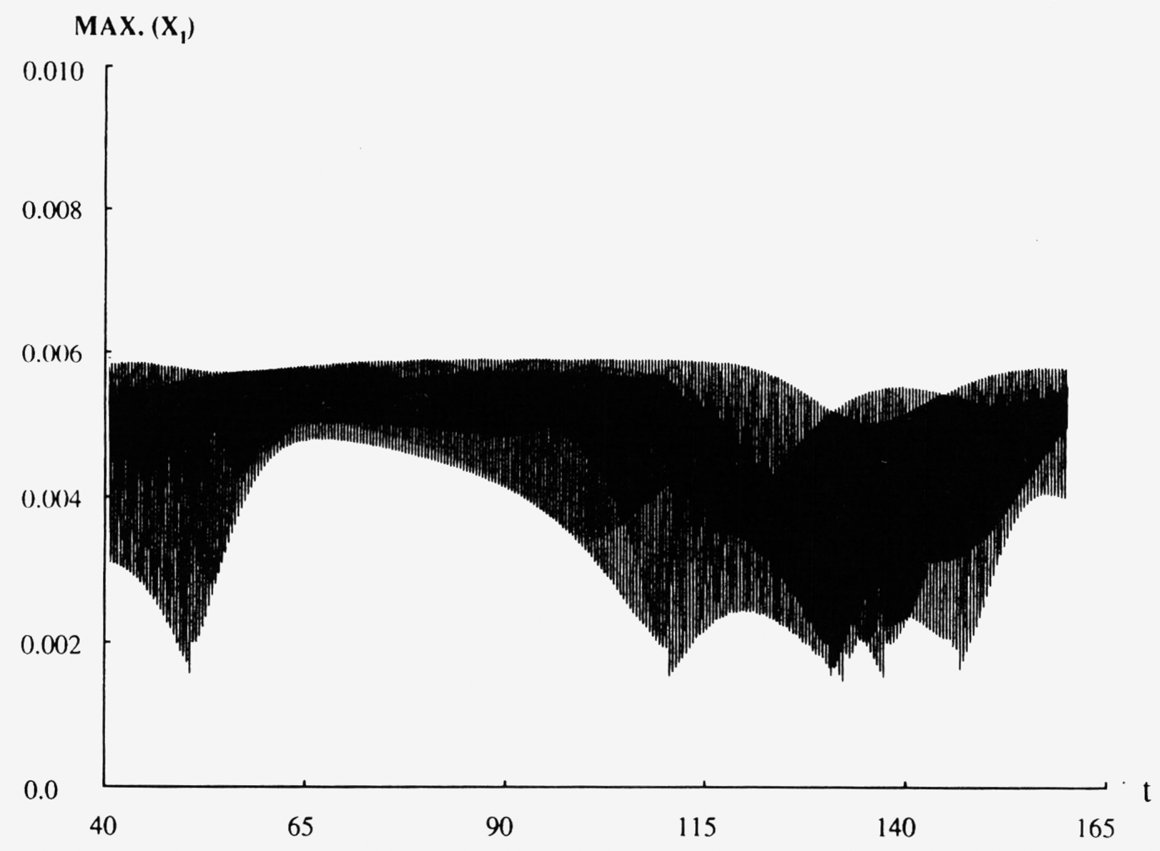

FIG. 3. $\max x_{1}$ for $40 \leq t \leq 163$. 


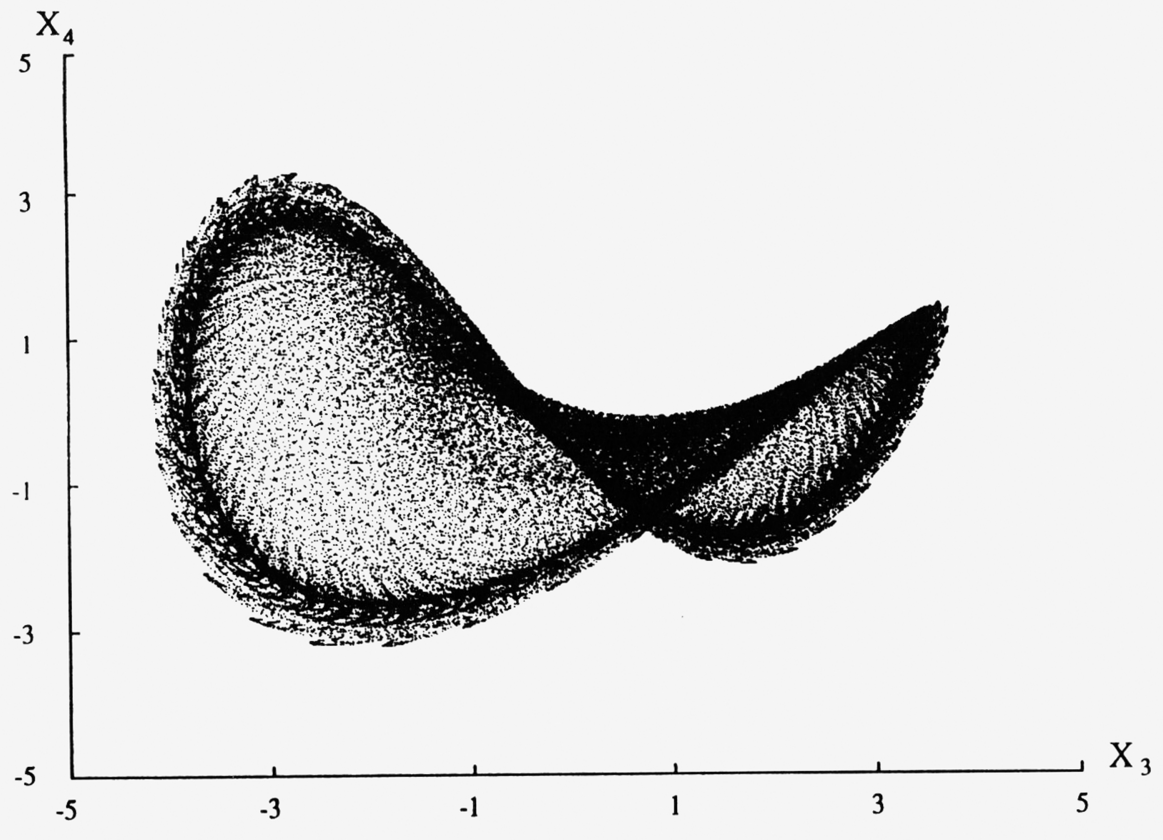

FIG. 4. $x_{4}$ vs. $x_{3}$ for $40 \leq t \leq 55$.

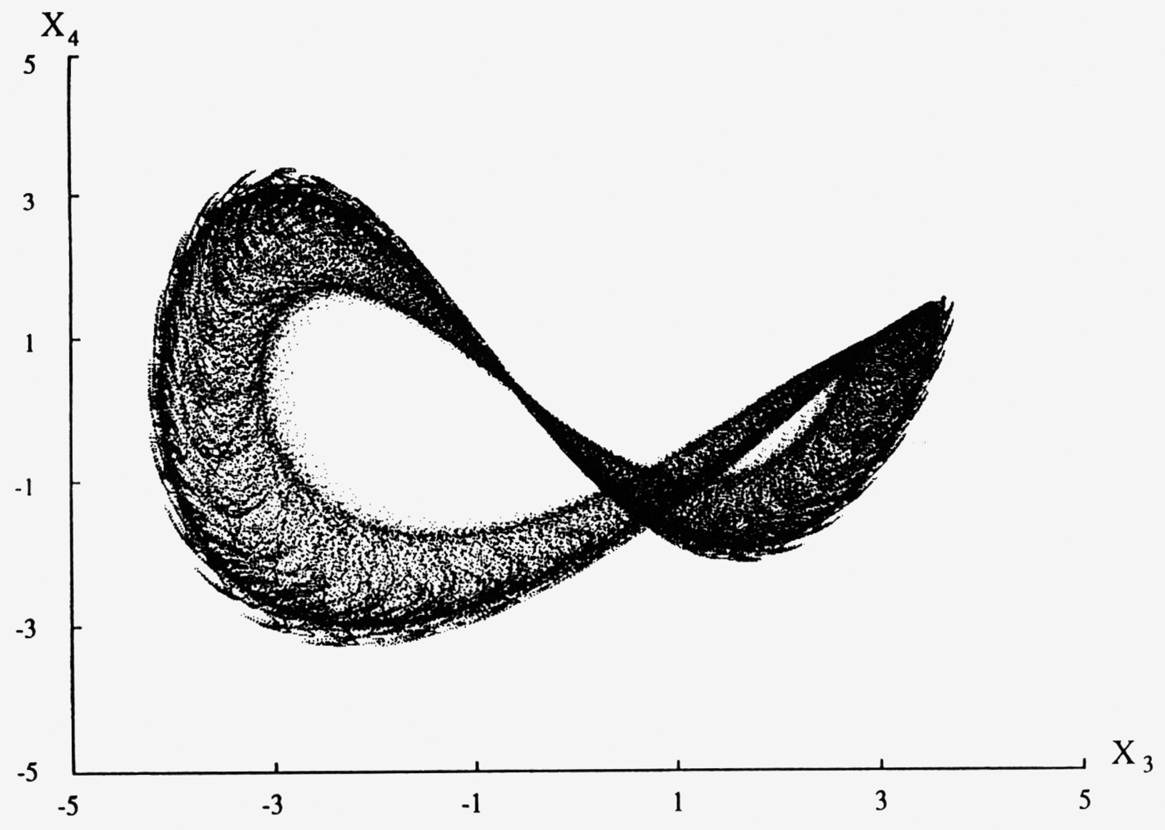

FIG. 5. $x_{4}$ vs. $x_{3}$ for $60 \leq t \leq 75$. 
606

B. S. BERGER, M. ROKNI, AND I. MINIS

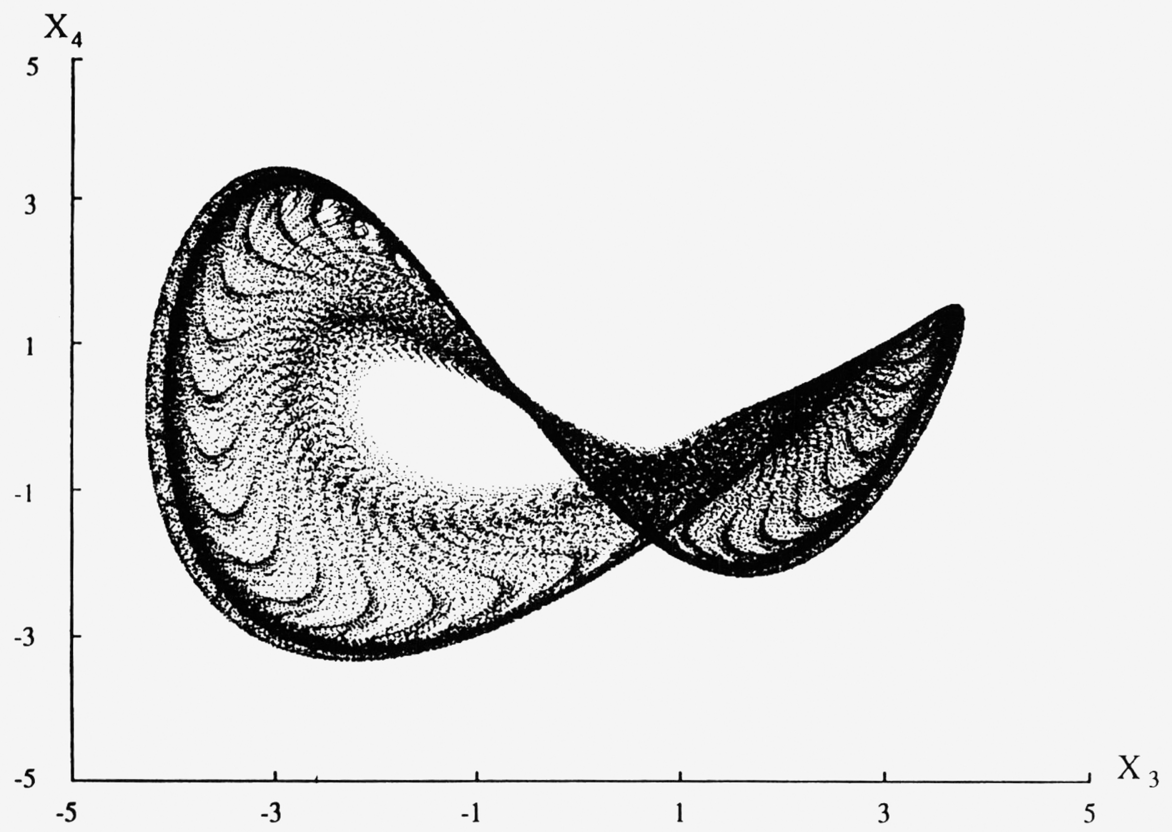

FIG. 6. $x_{4}$ vs. $x_{3}$ for $80 \leq t \leq 95$.

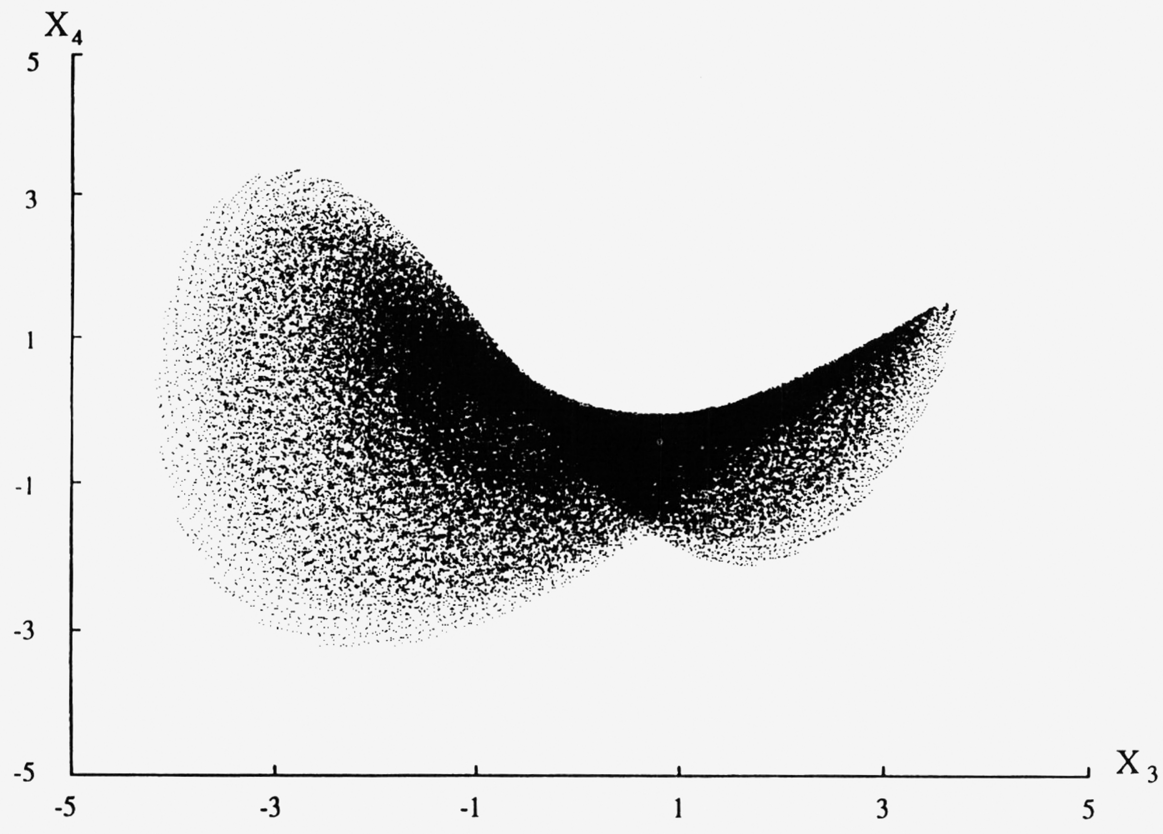

FIG. 7. $x_{4}$ vs. $x_{3}$ for $120 \leq t \leq 135$. 


$$
\underset{\infty}{\infty}
$$




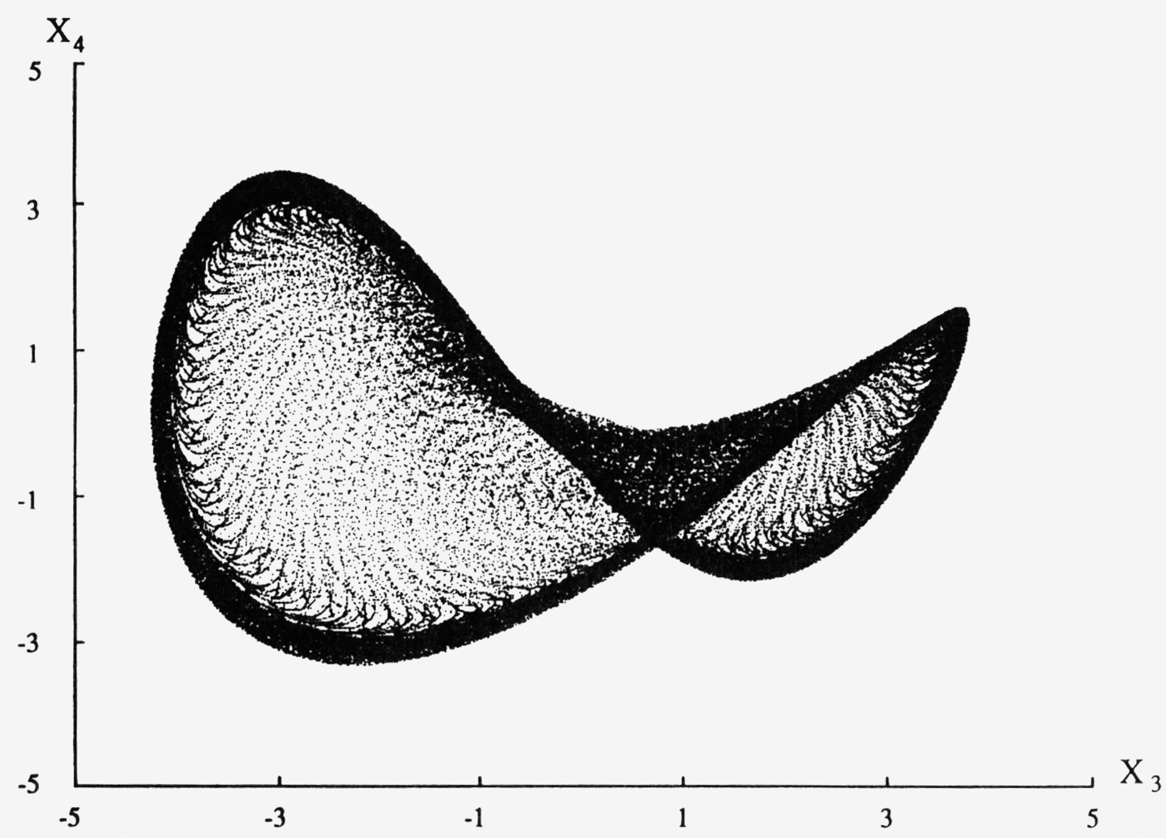

FIG. 10. $x_{4}$ vs. $x_{3}$ for $180 \leq t \leq 195$.

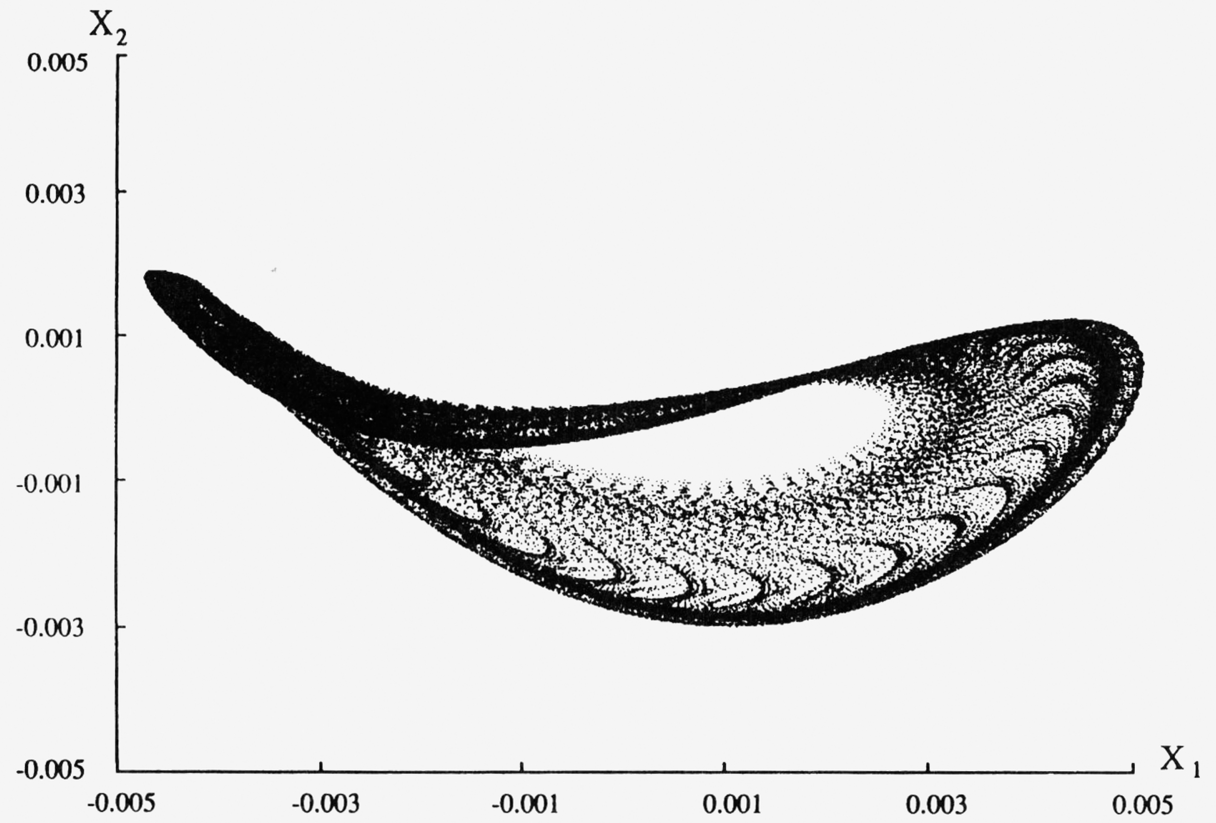

FIG. 11. $x_{2}$ vs. $x_{1}$ for $80 \leq t \leq 95$. 


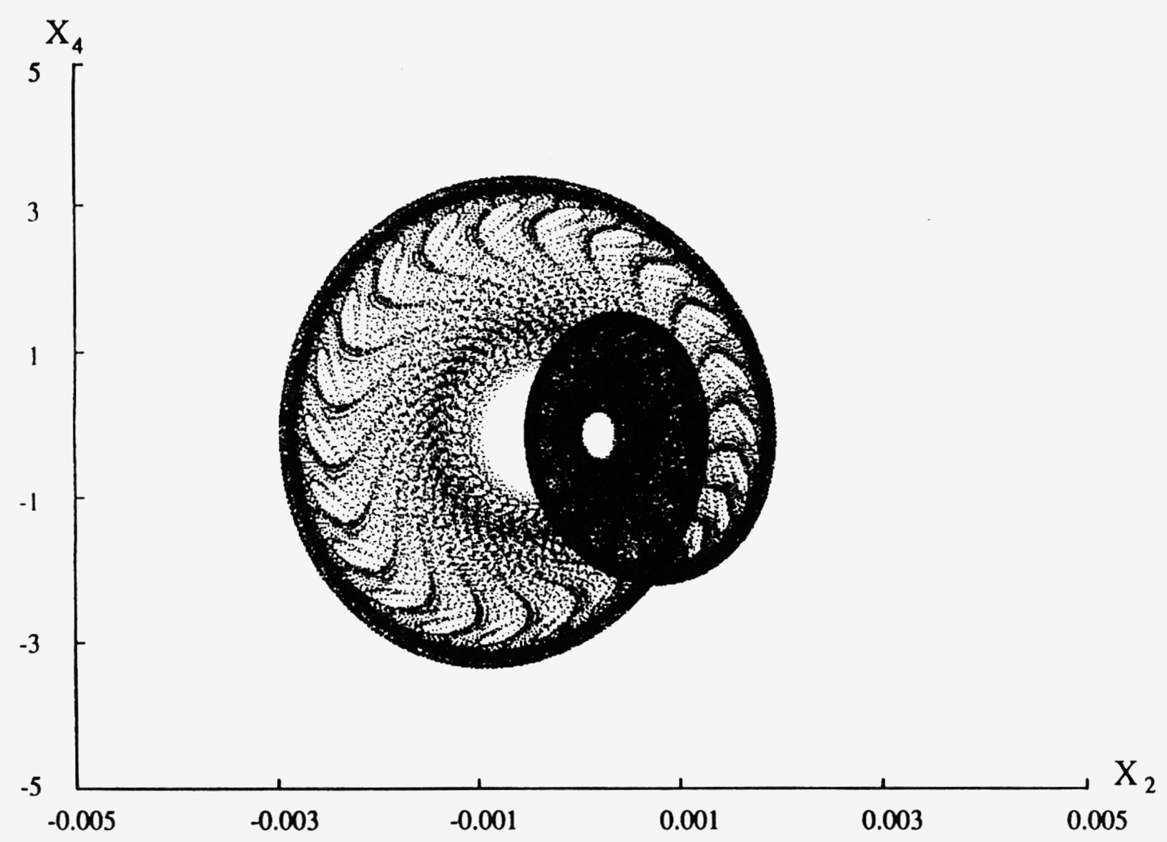

FIG. 12. $x_{4}$ vs. $x_{2}$ for $80 \leq t \leq 95$.

Dimension function. The dimension function $D(\gamma)$ for an attractor is defined as [10]

$$
D(\gamma)=-\lim _{n \rightarrow \infty} \log _{b}(n / k) /\left((1 / \gamma) \log _{b}(1 / m) \sum_{j=1}^{m} \delta_{j}^{\gamma}(k, E, n)\right)
$$

where $m \equiv$ number of reference points $\mathbf{y}_{j}, j=1, \ldots, m$, on the attractor, $E \equiv$ dimension of the embedding space, $\gamma=(1-q) D_{q}$ where $D_{q} \equiv$ Renyi dimension, $\delta_{j}^{\gamma}(k, E, n) \equiv$ distance between the reference points $\mathbf{y}_{j}$ and their $k$ th nearest neighbors for an $E$-dimensional embedding space, and $n \equiv$ number of generic data points. $D_{0}=$ capacity $D_{1}$, and $D(0)=$ information dimension. The parameter $\gamma$ weights large scales for $\gamma>0$ and small scales for $\gamma<0$. The variation of $D(\gamma)$, therefore, gives an estimate of the attractor's uniformity factor $\lambda$ [14]. The determination of $D(0)$ from Eq. (3), Fig. 13 (see p. 610), used a reconstruction of the attractor based on delaying $x_{1}$ by $i=1, \ldots, E, x_{i}(t) \equiv x_{1}(t+(i-1) \Delta t), m=3000, n=$ 477175 , and $k=200,300$. The estimated value of $D(0)=2.65 \pm 0.01$.

The pointwise mass dimension $D_{p}$ is defined as

$$
D_{p}=\lim _{n \rightarrow \infty} \lim _{r \rightarrow 0} \ln \left((1 / n) N\left(\mathbf{x}_{0}, r\right)\right) / \ln r
$$

where $N\left(\mathbf{x}_{0}, r\right) \equiv$ the number of attractor points within a cube of side length $r$ centered at point $\mathbf{x}_{0}$ on the attractor, and $n \equiv$ number of data points. $D_{p}$ is defined in terms of a fixed-size partition as contrasted with the fixed mass $D(\gamma)$. Computational limitations necessitate the approximation of the limits in Eq. (4) by an average over many reference points $\mathbf{X}_{i}$. The ratio $(\ln r) / \ln N\left(\mathbf{X}_{i}, r\right)$ is evaluated 


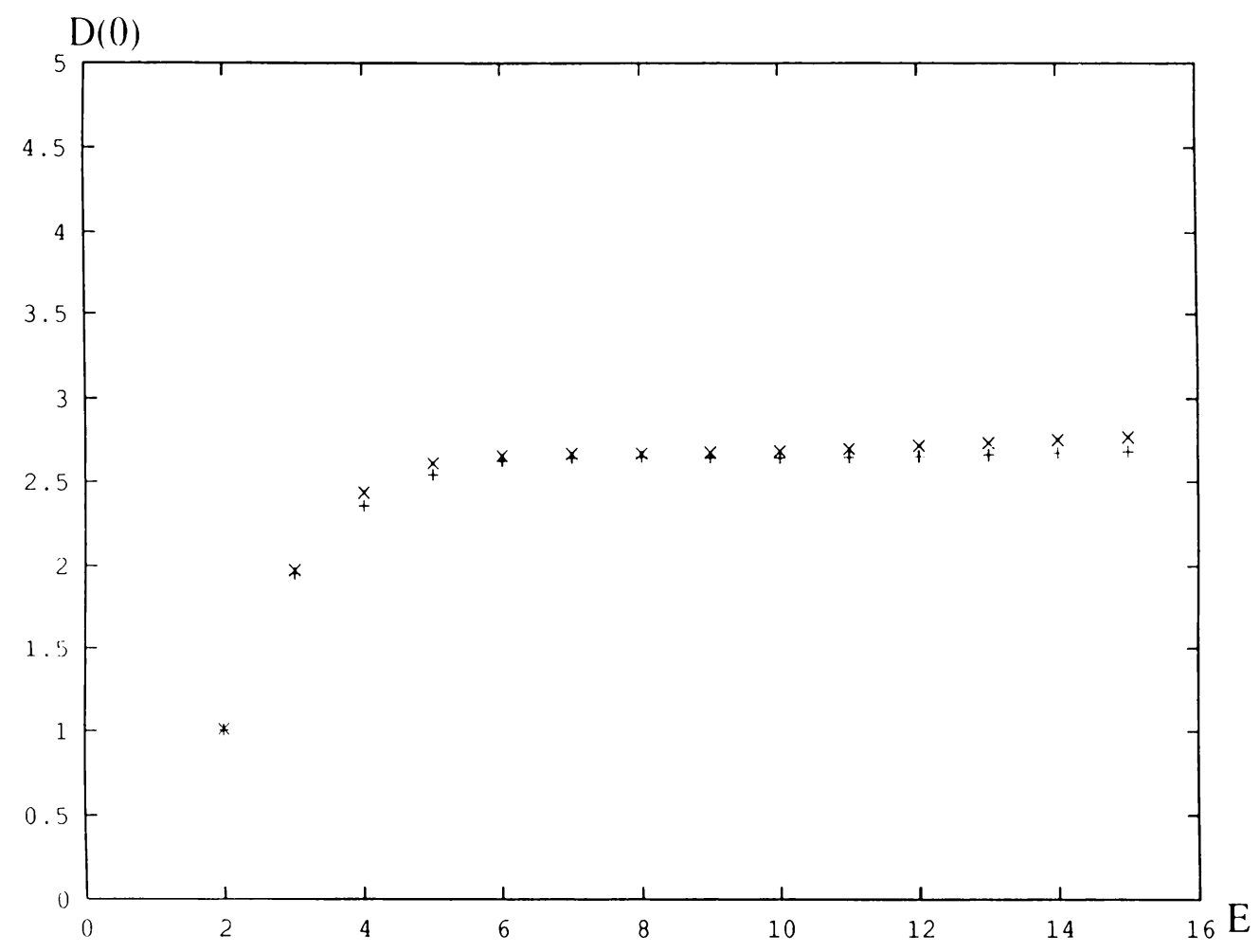

FIG. 13. $D(0)$ vs. $E$ for $E=1, \ldots, 15$.

at each $\mathbf{X}_{i}$ for a sequence of decreasing values of $r$ and approximated by a least square linear regression. Those $\mathbf{X}_{i}$ for which the estimated standard error of the ratio was $\leq 0.004$ for some scaling range in $r$ were retained in the average [10-12].

The infinite-dimensional differential-delay equation (2) possesses a finite-dimensional attractor. The Whitney embedding theorem for smooth manifolds implies that the unknown embedding dimension $M$ satisfies the inequality $M \leq 2 m+1$ where $m \equiv$ attractor dimension. Note that attractors associated with chaotic dynamics are not smooth manifolds. An asymptotic approximation of the attractor dimension was found by computing $D_{p}(E)$ for a sequence of increasing values of $E$ [13]. The average value of $D_{p}(E)=2.65 \pm 0.3$ for $2 \leq E \leq 5$ computed over 50,000 points. The values of $D_{p}$ and $D(0)$ are in reasonable agreement.

Metric entropy. The metric entropy $K(q)$ may be defined as

$$
K(q)=\lim _{\varepsilon \rightarrow 0} \lim _{E \rightarrow \infty}(1 /(E(1-q))) \ln \left(p^{q-1}\left(\varepsilon, Y_{j}(E)\right)\right)
$$

where $E \equiv$ dimension of the phase space and $P\left(\varepsilon, Y_{j}(E)\right) \equiv$ the probability of finding a generic embedded point $X_{i}(E)$ in an $\varepsilon$ neighborhood of $Y_{j}(E)$, a point of the embedding space. From Eq. (5) it follows that [15]

$$
\log _{\gamma \neq 0} \delta^{\gamma}(k, E, n) \sim \frac{\gamma}{D(\gamma)}\left[\Delta t \cdot E \cdot k(\gamma)-\log _{b} n\right]
$$




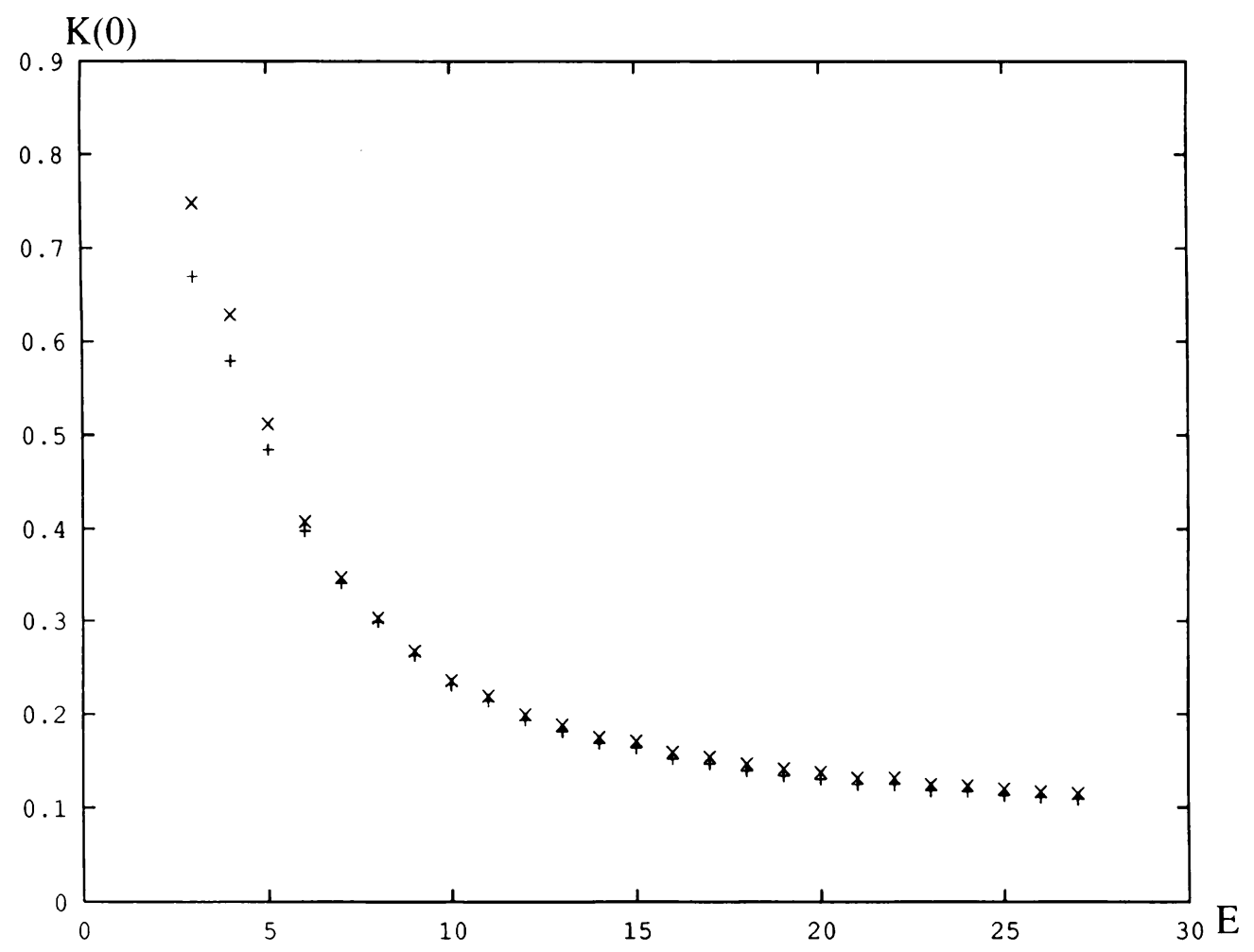

Fig. 14. $K$ vs. $E$ for $E=1, \ldots, 29$.

and

$$
\log _{\gamma=0} \delta(k, E, n) \sim \frac{1}{D(0)}\left[\Delta t \cdot E \cdot k(0)-\log _{b} n\right] .
$$

Quantities appearing in Eqs. (6) and (7) are as defined in Eq. (3). It is evident from Eqs. (6) and (7) that $K(0)$ and $K(\gamma)$ can be estimated by computing the mean difference between values of $\log _{b} \delta^{\gamma}(k, E, n)$ for pairs of phase space dimensions, $E_{r}$ and $E_{r+1}$. Figure 14 shows $K(0)$, the Kolmogorov metric entropy, for parameters identical to those used with $D(0)$. The estimated value of $K(0)=0.11, K(0) \leq$ the sum of the positive Lyapunov exponents $[5,16]$. It follows that the associated dynamics are chaotic for bounded $K(0)>0$.

Conclusions. A model of metal cutting, based on the Wu-Liu theory of cutting forces, has been shown to possess noninteger dimensions, positive metric entropy, and surface-filling trajectory geometry, all of which indicate the presence of chaotic dynamics. The geometry of trajectories on the attractor showed subharmonic, aperiodic evolution. It follows that the complex nature of regenerative metal-cutting vibrations may be a manifestation of chaotic dynamics.

Acknowledgments. The authors gratefully acknowledge the assistance of C. J. Berger, S. Woo, S. Austen, and P. Chen. Computer computations were supported by the Computer Science Center at the University of Maryland. Support was also received from D.O.E. through DE-FG02-93ER14335. 


\section{REFERENCES}

[1] I. Grabec, Chaos generated by the cutting process, Phys. Lett. A 117, 384-386 (1986)

[2] I. Grabec, Explanation of random vibrations in cutting on grounds of deterministic chaos, Robotics and Comp.--Integrated Manufacturing 4, 129-134 (1988)

[3] I. Grabec, Chaotic dynamics of the cutting process, Int. J. Mach. Tools Manufact. 28, 19-32 (1988)

[4] D. W. Wu and C. R. Liu, An analytical model of cutting dynamics, Parts 1 and 2, ASME J. Engrg. Indust. 17, 107-111, 112-118 (1985)

[5] J. D. Farmer, E. Ott, and J. A. Yorke, The dimension of chaotic attractors, Phys. D 7, 153-180 (1983)

[6] I. Minis, E. Magrab, and I. Pandelidis, Improved methods for the prediction of chatter in turning, Part 1, ASME J. Engrg. Indust. 112, 11-20 (1990)

[7] A. C. Eringen, Mechanics of Continua, Wiley, New York, 1967

[8] J. K. Hale, Theory of Functional Differential Equations, Springer-Verlag, New York, 1977

[9] P. J. Houwen and B. P. Sommeijer, Stability in linear multistep methods for pure delay equations, J. Comput. Appl. Math. 16, 55-63 (1984)

[10] G. Broggi, Evaluation of dimension and entropies of chaotic systems, J. Opt. Soc. Amer. B 5, 1020-1028 (1988)

[11] J. Holzfuss and G. Mayer-Kress, An approach to error-estimation in the application of dimension algorithms, Dimensions and Entropies in Chaotic Systems, G. Mayer-Kress, ed., Springer-Verlag, New York, 1985, pp. 114-122

[12] E. J. Kostelich and H. L. Swinney, Practical considerations in estimating dimension from time series data, Phys. Scripta 40, 436-441 (1989)

[13] J. D. Farmer, Order within chaos, Dissertation, Univ. of Calif., Santa Cruz, CA, 1981

[14] R. Badii and A. Politi, Statistical description of chaotic attractors: The dimension function, J. Statist. Phys. 40, 516, 725-750 (1985)

[15] P. Grassberger, Chaos, A. Y. Holden, ed., Manchester Univ. Press, Manchester, 1986

[16] J. P. Eckmann and D. Ruelle, Ergodic theory of chaos and strange attractors, Rev. Modern Phys. 57, 3 (1985) 